\title{
Safety and Effectiveness of Rituximab in Patients with Rheumatoid Arthritis Following an Inadequate Response to 1 Prior Tumor Necrosis Factor Inhibitor: The RESET Trial
}

\author{
BOULOS HARAOUI, MARIA BOKAREWA, IAN KALLMEYER, and VIVIAN P. BYKERK, for the RESET Investigators
}

\begin{abstract}
Objective. To evaluate the safety and effectiveness of rituximab (RTX) in combination with methotrexate in patients with active rheumatoid arthritis (RA) after failure of a single tumor necrosis factor- $\alpha$ $(\mathrm{TNF}-\alpha)$ inhibitor. Changes in patient-reported outcomes after primary treatment or retreatment with RTX and factors determining retreatment in clinical practice were also evaluated.

Methods. In this phase $3 \mathrm{~b}$ open-label, multicenter trial, patients received 2 slow infusions of RTX 1000 mg 14 days apart after premedication (primary treatment). Patients with a clinically relevant response could receive retreatment between 24 and 48 weeks. The primary endpoint was evaluation of safety. Secondary outcomes were safety of retreatment, effectiveness of primary treatment and retreatment, and changes in patient-reported outcomes after primary treatment or retreatment.

Results. Of 120 patients enrolled at 36 centers and receiving primary RTX treatment, 77 received retreatment, 112 completed the 24 -week primary treatment period, and 25 completed the 48 -week primary treatment and retreatment period following a single course of RTX. The most common adverse events were mild to moderate nausea, vomiting, nasopharyngitis, and headache. No infections or infusion reactions were considered life-threatening. At 24 weeks, 58\%, 27\%, and 7\% of patients achieved American College of Rheumatology 20, 50, and 70 improvements, respectively, and similar improvements were seen after retreatment.

Conclusion. RTX was well tolerated, with a low incidence of infusion reactions and infections. Efficacy results, including enhanced response in rheumatoid factor-positive patients, were comparable to those reported in the literature. Based on its efficacy and safety profile and retreatment schedule, RTX is an attractive treatment option for patients that have not responded to a single TNF- $\alpha$ inhibitor. (First Release Oct 1 2011; J Rheumatol 2011;38:2548-56; doi:10.3899/jrheum.110444)
\end{abstract}

\section{Key Indexing Terms: \\ RHEUMATOID ARTHRITIS \\ ANTI-CD20 ANTIGEN}

$\begin{array}{lll}\text { RITUXIMAB MONOCLONAL ANTIBODY } & \text { MATS } \\ \text { TUMOR NECROSIS FACTOR INHIBITOR }\end{array}$
Rheumatoid arthritis (RA) is a systemic inflammatory disease characterized by chronic inflammation of the synovial membrane, primarily affecting the small joints of the hands and feet ${ }^{1}$. About $55 \%$ to $70 \%$ of patients experience progressive deterioration of cartilage and bone in the affected joints, resulting in deformity and chronic pain. Most patients with

From the University of Montreal, Montreal, Quebec, Canada; the University of Gothenburg, Gothenburg, Sweden; Department of Medical and Regulatory Affairs, Hoffmann-La Roche Ltd., Mississauga, Ontario; and the University of Toronto, Toronto, Ontario, Canada.

Funded by Hoffmann-La Roche Canada and Roche Sweden, AB. B. Haraoui, MD, FRCPC, Clinical Associate Professor of Medicine, University of Montreal; M. Bokarewa, MD, Associate Professor, University of Gothenburg; I. Kallmeyer, BSc, Director, Department of Medical and Regulatory Affairs, Hoffmann-La Roche Ltd.; V.P. Bykerk, MD, FRCPC, Assistant Professor of Medicine, University of Toronto.

Address correspondence to Dr. B. Haraoui, Institut de rhumatologie de Montréal, 1551 Ontario est, Montréal, Quebec H2L 1S6, Canada.

E-mail: boulos.haraoui@ssss.gouv.qc.ca

Full Release Article. For details see Reprints/Permissions at jrheum.org Accepted for publication July 20, 2011.
RA eventually experience functional decline and work disability and on average have a lower life expectancy compared with the general population ${ }^{2}$.

Over the past 10 years, the advent of tumor necrosis factor- $\alpha$ (TNF- $\alpha$ ) inhibitors has been a significant advance in the management of RA. However, about $25 \%$ of patients treated with TNF- $\alpha$ inhibitors either do not receive adequate clinical benefit or are unable to tolerate them during the first year, and more patients lose efficacy over time ${ }^{3}$. Other data suggest that treatment with an additional TNF- $\alpha$ inhibitor may lead to progressively diminishing effectiveness over time ${ }^{4}$. Several well controlled clinical trials have investigated the efficacy of newer biologics other than rituximab (RTX) after the failure of TNF- $\alpha$ inhibitors ${ }^{5,6,7}$. The results of those trials provide additional evidence and therefore treatment options when there is no response to the initial TNF- $\alpha$ inhibitor.

RTX, a chimeric monoclonal antibody that selectively depletes B cells expressing the CD20 antigen, is a biologic agent indicated for use after the failure of a TNF- $\alpha$ inhibitor ${ }^{8}$.

Personal non-commercial use only. The Journal of Rheumatology Copyright $($ C 2011. All rights reserved. 
B cells have been shown to play a central role in the pathogenesis of RA and may have multiple actions in the autoimmune and inflammatory processes, including production of rheumatoid factor (RF), other autoantibodies, and proinflammatory cytokines, as well as antigen presentation and $\mathrm{T}$ cell activation. Several controlled phase 2 and 3 trials have investigated the combination of RTX plus methotrexate (MTX) in patients with RA whose disease responded inadequately to MTX alone $9,10,11,12$. One study evaluated the efficacy and safety of RTX in patients naive to $\mathrm{MTX}^{13}$. An additional phase 3 study investigated RTX in cases with an inadequate response to 1 or more TNF- $\alpha$ inhibitors ${ }^{14}$. Longterm followup of patients treated with RTX in clinical trials has demonstrated good tolerability over multiple courses, with a safety profile similar to that of the placebo population ${ }^{15}$. Moreover, sustained clinical efficacy has also been documented over repeated courses ${ }^{16,17}$.

The introduction of RTX and other non-TNF- $\alpha$ biologic agents has raised the question of the sequence in which they should be used relative to an inadequate response to TNF- $\alpha$ inhibitors. Our study was designed to evaluate the safety and effectiveness of RTX in combination with MTX in patients with RA after failure of a single TNF- $\alpha$ inhibitor. We also evaluated changes in patient-reported outcomes following primary treatment or retreatment with RTX and gathered data on the factors determining retreatment with RTX in routine clinical practice.

\section{MATERIALS AND METHODS}

Patients. One hundred twenty patients were recruited from 36 sites in Canada and Sweden. Patients were included if they were age 18 to 80 years; had been treated with MTX 10 to $25 \mathrm{mg} /$ week for at least 12 weeks prior to screening and at a stable dose for at least 4 weeks before study entry; had experienced the failure of a single TNF- $\alpha$ inhibitor because of toxicity or inefficacy (inefficacy was left to the discretion of the investigator as long as the patient had an adequate trial of a TNF- $\alpha$ inhibitor: $\geq 3$ months at $25 \mathrm{mg}$ twice a week or $50 \mathrm{mg}$ weekly for etanercept; at least 3 infusions of infliximab at $\geq 3 \mathrm{mg} / \mathrm{kg}$; or $\geq 3$ months at $40 \mathrm{mg}$ every other week for adalimumab); and documented moderate to severe active RA, with at least 6 tender and 6 swollen joints with an elevated C-reactive protein (CRP) or erythrocyte sedimentation rate (ESR) or morning stiffness lasting at least $30 \mathrm{~min}$. Subjects of reproductive potential had to use a reliable means of contraception, and women of childbearing potential had to have a negative serum pregnancy test. For retreatment, patients had to have active RA, defined as a 28 -joint disease activity score (DAS28) > 2.6, at least 24 weeks after the initial RTX infusion.

Patients were excluded if they had functional stage IV disability, had undergone bone or joint surgery within 8 weeks of Day 1 or had joint surgery planned within 48 weeks, had a history of severe allergic or anaphylactic reactions to monoclonal antibodies, or had any severe or significant medical condition or disease that, in the view of the investigator, should prohibit them from participation in the study, including significant cardiac or pulmonary disease, known active infection or history of serious recurrent or chronic infection, a major episode of infection requiring hospitalization or treatment with an intravenous (IV) antiinfective within 4 weeks, or an oral antiinfective within 2 weeks of study entry. Patients could not have immunodeficiency, be pregnant or lactating, or have a history of malignancy, except excised basal cell or squamous cell carcinoma of the skin. Subjects were not included if they had had previous treatment with RTX or any other cell-depleting therapy, or etanercept within 4 weeks, or adalimumab or infliximab within 8 weeks of first RTX treatment. Subjects could not have had a vaccination or intraarticular or parenteral glucocorticoids within 4 weeks of study entry. Treatment with any investigational agent within 4 weeks of study entry or 5 half-lives, whichever was longer, was not permitted.

Concomitant medications. Concurrent treatment with a corticosteroid such as prednisone, not $>10 \mathrm{mg} /$ day, was permitted if the dose was stable for at least 4 weeks before study entry. Concurrent use of nonsteroidal antiinflammatory drugs was permitted if the dose was stable for at least 2 weeks before Day 1 . Acetylsalicylic acid, not $>325 \mathrm{mg} / \mathrm{day}$, was allowed for cardiovascular prophylaxis. The only concomitant disease-modifying antirheumatic drug therapy permitted was MTX, with dosage modification allowed for toxicity or RA flare. Analgesics were permitted for pain as required but prohibited within 24 $\mathrm{h}$ of any visit in which clinical efficacy was assessed. Intraarticular corticosteroids were limited to only 1 joint per 24-week period; the same joint could not be injected twice. An increased dose of any RA therapy was regarded as an adverse event (AE). TNF- $\alpha$ inhibitors and other biologic therapies were prohibited; patients requiring such medication were withdrawn from the study and entered into the safety followup period.

The study was conducted in conformance with the principles of the Declaration of Helsinki and local regulations. All patients provided written informed consent.

Study protocol. This phase 3b, open-label, multicenter trial (registration number NCT01272908) evaluated the safety and effectiveness of RTX in patients with RA after failure of a single previous TNF- $\alpha$ inhibitor. Patients who met the eligibility criteria were enrolled to receive RTX 1000 mg IV on Days 1 and 15, in addition to concomitant MTX therapy. All patients also received folic acid, at least $5 \mathrm{mg} / \mathrm{week}$, during the entire trial.

All patients received premedication with a slow infusion of methylprednisolone, $100 \mathrm{mg} \mathrm{IV}$, at least $30 \mathrm{~min}$ prior to each infusion of RTX, to reduce the incidence and severity of infusion reactions ${ }^{8}$. The following premedication was recommended 30 to $60 \mathrm{~min}$ before RTX infusion: acetaminophen, 1 $\mathrm{g}$ orally, and diphenhydramine hydrochloride, $50 \mathrm{mg}$ orally, or IV equivalent, or equivalent dose of a similar antihistamine.

Patients achieving a clinically relevant response (defined by the investigator) to the primary treatment who had a DAS28 score $>2.6$ could receive retreatment between 24 and 48 weeks at the discretion of the investigator. Retreatment consisted of 2 RTX infusions, $1000 \mathrm{mg}$ IV, given 14 days apart. Modification of the RTX dose was not permitted.

Study objectives and endpoints. The primary study objective was evaluation of the safety of RTX in patients with active RA who were receiving MTX and had experienced treatment failure with a single TNF- $\alpha$ inhibitor. Safety was measured by the overall incidence of $\mathrm{AE}$, including infusion-related $\mathrm{AE}$ and infections, graded by severity and relationship to study drug on Days 1 and 15 , and at $4,8,12,24,36$, and 48 weeks after the primary treatment.

The secondary study objectives were evaluation of (1) the safety of retreatment with RTX in combination with MTX, (2) the effectiveness of primary treatment and retreatment with RTX in combination with MTX, and (3) changes in patient-reported outcomes following the primary treatment or retreatment. The safety of retreatment with RTX was measured by the overall incidence of $\mathrm{AE}$, including infusion-related $\mathrm{AE}$ and infections, graded by severity and relationship to the study drug, at $4,8,12$, and 24 weeks after the first retreatment dose. Effectiveness endpoints were evaluated at 4, 12, 24, 36, and 48 weeks after the initial RTX treatment and at 12 and 24 weeks of the retreatment period.

Exploratory endpoints included the timing of retreatment with RTX in routine patient care; the factors influencing the clinical decision to re-treat with RTX, including DAS28, DAS core component values, American College of Rheumatology (ACR) core component values, the presence or absence of disease flare at the time of retreatment, and additional clinical or laboratory factors leading to retreatment; and any correlation between the type of TNF- $\alpha$ inhibitor treatment failure and RTX response.

Assessments. Safety evaluation included use of concomitant medications, assessment of vital signs, physical examination, and hematology (complete blood count, hemoglobin, and absolute neutrophil count), immunology (quan-

Personal non-commercial use only. The Journal of Rheumatology Copyright $(\subset$ 2011. All rights reserved. 
titative $\operatorname{IgG}$, IgM, and IgA levels), and blood chemistry (alanine aminotransferase, aspartate aminotransferase, and alkaline phosphatase) throughout the study.

All AE identified during the study were recorded and described by regulatory seriousness criteria ${ }^{18}$, if applicable: duration and severity; suspected relationship to RTX; need for treatment; and management were described. Early identification and treatment of infection was a priority. Infusion reactions were defined as AE occurring during or within $24 \mathrm{~h}$ after an infusion and were treated according to severity.

Effectiveness assessments after the primary RTX treatment included the proportion of patients meeting ACR criteria for improvement of $20 \%$ (ACR20), 50\% (ACR50), and 70\% (ACR70); changes from baseline in each of the ACR core components [swollen joint count (SJC), tender joint count (TJC), physician's global assessment of disease activity, patient's global assessment of disease activity, patient's assessment of pain, Health Assessment Questionnaire-Disease Index (HAQ-DI), and ESR/CRP]; European League Against Rheumatism (EULAR) response rates and the proportion of patients with disease in remission according to DAS28; changes from baseline in DAS28 and each of the DAS28 core components (SJC and TJC, patient's global assessment of disease activity, and ESR); and changes from baseline in the Functional Assessment of Chronic Illness Therapy-Fatigue (FACIT-F) scale (0 to 52).

The effectiveness of RTX retreatment was measured by ACR20, 50, and 70; EULAR response rates; changes from baseline of DAS28, core components of the DAS28, core components of the ACR, HAQ, and the FACIT-F scale; and the proportion of patients with disease in remission according to DAS28.

Statistical analysis. All analyses used the intent to treat (ITT) population and SAS software (version 8.2 or higher). Frequencies and percentages were provided for all categorical measures. Continuous variables were summarized by number of patients, mean, SD, median, minimum, and maximum. Logistic regression analysis was conducted for patients meeting the ACR20, ACR50, and ACR70 criteria and the EULAR response criteria. An analysis of change from baseline was performed on the ACR and DAS28 core components. Efficacy variables (ACR, DAS28, and HAQ-DI) were also evaluated by baseline RF status. A paired t test or Wilcoxon signed-rank test was used to obtain the $p$ value, depending on normality of the data. An analysis of change from baseline was conducted for the total FACIT-F scale score and summarized using descriptive statistics. An interim analysis of safety and effectiveness was performed after about half the study population completed the 24-week study visit.

The proportion of patients with disease in remission at each visit according to DAS28 was summarized. Disease remission and duration of remission were derived from the DAS28 score. The timing of RTX retreatment was computed for each patient and summarized descriptively. Factors involved in the clinical decision for RTX retreatment and response to RTX based on the type of TNF- $\alpha$ inhibitor treatment failure were summarized in frequency tables. The number of patients meeting ACR20, ACR50, and ACR70 criteria, as well as a good or moderate EULAR response, was tabulated according to the category of TNF- $\alpha$ inhibitor treatment failure.

The primary imputation method for patients with missing effectiveness data was the nonresponder method, in which all missing data were considered as nonresponses. Last observation carried forward (LOCF) imputation was used for swollen and tender joint counts. Patients entering the safety followup period were classified as premature withdrawals and their values for effectiveness measures were imputed using LOCF. Analyses of safety and tolerability used the ITT population. All AE recorded were summarized and AE leading to study discontinuation were also listed separately. All events were coded using the Medical Dictionary for Regulatory Activities (MedDRA), version 11.1 and 12.0, and were graded according to Common Terminology Criteria for Adverse Events (CTCAE v 3.0).

The protocol was designed in consultation with the primary clinical investigators

\section{RESULTS}

Patient disposition. Overall, 120 patients were enrolled in
Canada $(\mathrm{n}=91)$ and Sweden $(\mathrm{n}=29)$ at 36 centers (Figure 1). One hundred twelve patients (93.3\%) completed the 24-week primary treatment period, and 25 patients $(20.8 \%)$ completed the 48-week primary treatment period (24-week treatment and 24-week followup periods) after receiving only a single course of RTX. Ten patients were assessed for retreatment after completing the 48 -week primary treatment period; 8 received retreatment and 2 withdrew without receiving retreatment.

Of the 77 patients $(64.2 \%)$ who received retreatment between Weeks 24 and 48, 72 patients (93.5\%) completed the 24-week retreatment period and 6 patients $(7.8 \%)$ withdrew (1 patient completed the retreatment screening visit but did not enter the retreatment period. The patient withdrew prior to receiving the drug).

The most common reason for study discontinuation, insufficient therapeutic response, resulted in withdrawal of 8 patients $(6.7 \%), 5$ during the primary treatment period and 3 during retreatment. Other reasons for discontinuation were $\mathrm{AE}$ in 3 patients $(2.5 \%)$, withdrawal of consent in 2 patients $(1.7 \%)$, and violation of entry criteria in 1 patient $(0.8 \%)$.

Patient characteristics. Table 1 summarizes the baseline patient demographics and disease characteristics for the overall population as well as the Canadian and Swedish cohorts. Despite slightly shorter disease duration, patients from Canada had more clinically severe and active disease relative to those from Sweden.

Safety. Overall, 120 patients received a mean RTX dose of $1977.1 \mathrm{mg}$ during the primary treatment period, and 77 patients received a mean RTX dose of $2000 \mathrm{mg}$ during the retreatment period. During the primary treatment period, 102 patients $(85 \%)$ experienced $512 \mathrm{AE}$, of which only $30 \%$ were considered to be treatment-related, and during retreatment, 53 patients (69\%) experienced $150 \mathrm{AE}$, of which $29 \%$ were considered to be treatment-related (Table 2). The most commonly reported AE were nausea, nasopharyngitis, headache, and vomiting, which were mild or moderate in intensity. Two life-threatening (grade 4) events were reported during each treatment period (neutropenia and RA during primary treatment, and large intestine perforation and ovarian epithelial cancer during retreatment), but none was considered related to the study drug. Twenty-one severe (grade 3) events were reported during the primary treatment period. Of those, 5 were considered to be related to treatment (breast cellulitis, headache and RA, pneumonia, dyspepsia, and urinary tract infection). Twelve patients experienced 14 serious adverse events (SAE) during primary treatment, and 7 patients reported 8 SAE during retreatment. Overall, 3 patients experienced AE leading to withdrawal. No deaths were reported during our study.

During primary treatment, 57 infections were reported; the most common infections ( $\geq 5 \%$ of patients) were nasopharyngitis $(10.8 \%)$, urinary tract infection $(7.5 \%)$, upper respiratory tract infection (5.8\%), sinusitis (5.0\%), and bronchitis (5.0\%). During retreatment, 21 infections were reported; the most

Personal non-commercial use only. The Journal of Rheumatology Copyright @ 2011 . All rights reserved. 


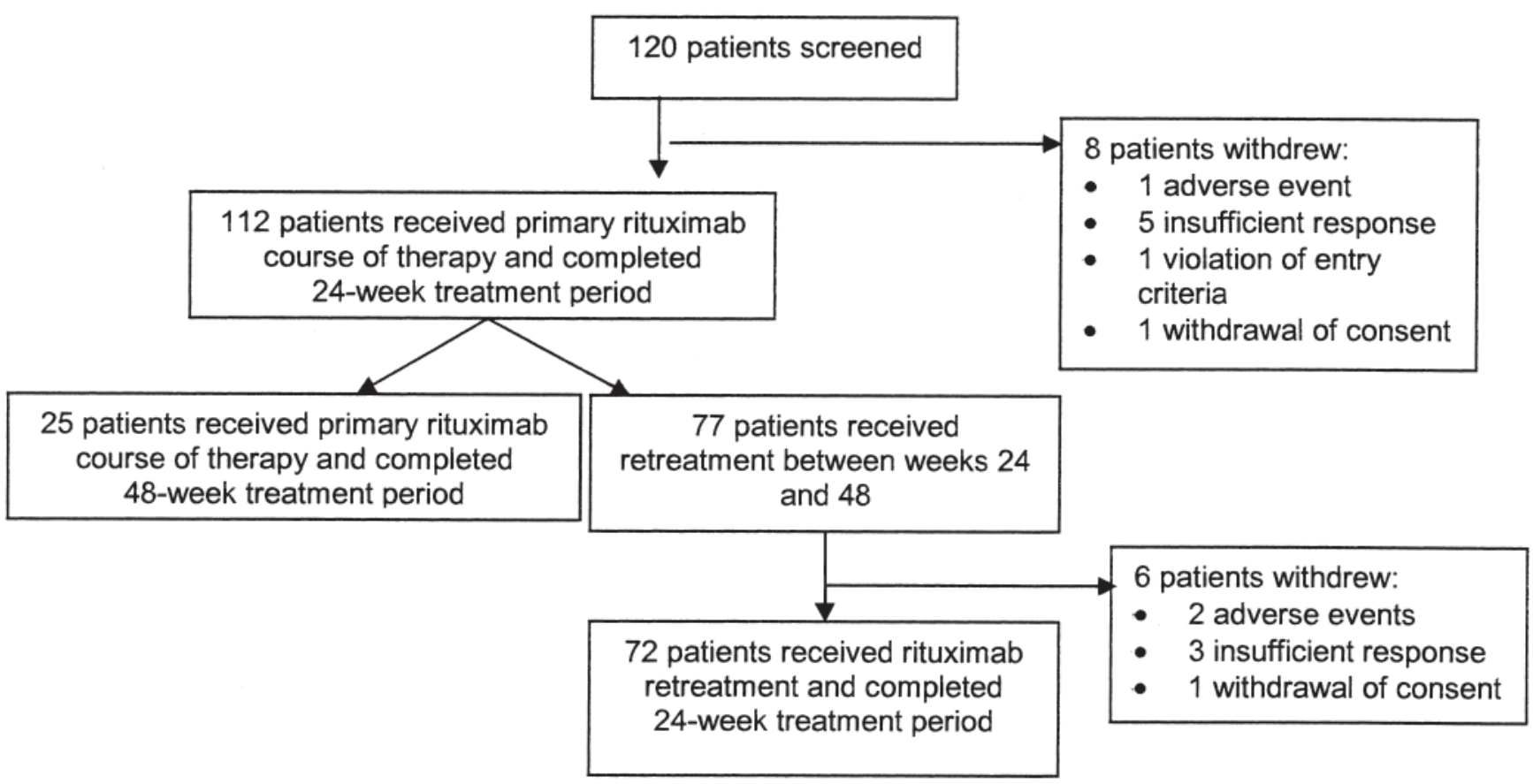

Figure 1. Patient disposition after primary treatment and retreatment. One patient completed the retreatment screening visit but did not enter the retreatment period. Patient withdrew prior to receiving drug.

Table 1. Patient demographics and baseline disease characteristics: intent-to-treat population.

\begin{tabular}{lccc}
\hline Characteristics & Overall, $\mathrm{n}=120$ & Canada, $\mathrm{n}=91$ & Sweden, $\mathrm{n}=29$ \\
\hline Age, yrs, mean \pm SD & $55.6 \pm 10.20$ & $54.8 \pm 9.59$ & $58.3 \pm 11.70$ \\
Men/women, $\%$ & $27.5 / 72.5$ & $25.3 / 74.7$ & $34.5 / 65.5$ \\
Race, $\mathrm{n}$ & & & \\
White & 113 & 84 & 29 \\
Black & 1 & 1 & 0 \\
Asian & 1 & 1 & 0 \\
Other & 5 & 5 & 0 \\
RA duration, yrs, mean \pm SD & $13.9 \pm 9.78$ & $13.6 \pm 8.96$ & $15.2 \pm 12.09$ \\
TJC, mean \pm SD & $16.1 \pm 6.62$ & $17.4 \pm 6.66$ & $12.0 \pm 4.59$ \\
SJC, mean \pm SD & $13.7 \pm 5.33$ & $14.4 \pm 5.40$ & $11.5 \pm 4.54$ \\
DAS28, mean \pm SD & $6.4 \pm 1.06$ & $6.5 \pm 1.10$ & $6.2 \pm 0.93$ \\
HAQ-DI, mean \pm SD & $1.7 \pm 0.57$ & $1.8 \pm 0.55$ & $1.4 \pm 0.51$ \\
Global assessment of pain, mean \pm SD & $60.2 \pm 22.85$ & $62.6 \pm 22.83$ & $52.8 \pm 21.62$ \\
Global assessment of disease activity, mean + SD & $67.5 \pm 22.73$ & $69.0 \pm 23.57$ & $62.8 \pm 22.32$ \\
FACIT-F, mean \pm SD & $28.9 \pm 10.22$ & $30.1 \pm 10.52$ & $25.2 \pm 8.31$ \\
RF+, $n(\%)$ & $89(74.2)$ & $66(72.5)$ & $23(79.3)$ \\
CRP, mg/dl & 25.6 & 24.2 & 30.1 \\
ESR, mm/h & 37.0 & 34.5 & 44.8 \\
\hline
\end{tabular}

CRP: C-reactive protein; DAS28: 28-joint count Disease Activity Score; HAQ-DI: Health Assessment Questionnaire Disability Index; RA: rheumatoid arthritis; RF+: rheumatoid factor-positive; SJC: swollen joint count; TJC: tender joint count; ESR: erythrocyte sedimentation rate; FACIT-F: Functional Assessment of Chronic Illness Therapy Fatigue.

common were nasopharyngitis $(10.4 \%)$, upper respiratory tract infection (3.9\%), and urinary tract infection (3.9\%). The serious infection rate per 100 patient-years was 3.8 over the 48-week primary treatment period and 5.8 following 24 weeks during the retreatment period. Most infections occurring during the study were mild or moderate. Of 5 severe infections during primary treatment, 3 were considered to be treatment-related ( 2 cases of pneumonia and 1 case of urinary tract infection). One case of treatment-related breast cellulitis during the retreatment period was reported as a severe infection. No life-threatening infections (grade 4) occurred during the study. 
Table 2. Overview of adverse events (AE).

\begin{tabular}{lcc}
\hline & $\begin{array}{c}\text { Primary Treatment*, } \\
\mathrm{n}=120(\%)\end{array}$ & $\begin{array}{c}\text { Retreatment }^{\dagger}, \\
\mathrm{n}=77(\%)\end{array}$ \\
\hline Patients with AE & $102(85)$ & $53(69)$ \\
Total AE & 512 & 150 \\
Treatment-related AE/SAE, $\%$ & 30 & 29 \\
Patients with AE leading & $12(10)^{* * *}$ & $2(2.6)^{\dagger \dagger}$ \\
$\quad$ to withdrawal & 0 & $7(9.1)^{\#}$ \\
Patients with SAE & & 0 \\
Deaths & $12 *$ & \\
\hline
\end{tabular}

* Weeks 0-48. ${ }^{\dagger}$ Weeks $0-24 . * *$ AE/SAE leading to withdrawal (all determined to be unrelated unless noted): herpes zoster (related). ${ }^{\dagger}$ Prostate cancer, pneumonia. *** Serious adverse event (SAE): chest pain, labyrinthitis, pneumonia (related), urinary tract infection (related), prostate cancer, RA flare, transient ischemic attack, neutropenia, abdominal discomfort, uterine leiomyoma, breast cancer, hip fracture. \# Vision blurred, large intestine perforation, breast cellulitis (related), upper limb fracture, headache (related), ovarian epithelial cancer, urinary tract infection and pulmonary fibrosis (same patient).

Infusion reactions occurred in 25 patients $(20.8 \%)$ during primary treatment and 10 patients $(13.0 \%)$ during retreatment. The most frequent treatment-related infusion reactions were headache, nausea, pruritus, and dizziness. No infusion reaction was serious or resulted in discontinuation of dosing. Headache, the only severe reaction reported, occurred in 1 patient.

No clinically significant changes occurred in laboratory test results during the study. Specifically, no significant changes from baseline were seen in $\operatorname{IgG}$, IgM, or IgA during treatment or retreatment. No clinically meaningful changes occurred in vital signs measurements (heart rate, arterial pressure, and temperature); minor changes seen during RTX infusion returned to pre-dose levels during the postinfusion observation period.

Effectiveness. At 24 weeks of primary treatment, 58\%, 27\%, and $7 \%$ of the population achieved ACR20, 50, and 70 improvements, respectively (Figure 2A). A greater proportion of RF-positive than RF-negative patients showed improvement at Week 24 (Figure 2B). The number of patients with ACR50 responses increased significantly at 12 weeks $(\mathrm{p}=$ $0.023)$ and 24 weeks $(p=0.001)$, compared with the 4-week improvement. Among RF-positive patients, significant increases were seen in ACR20 at 24 weeks $(\mathrm{p}=0.006)$ and ACR50 at 12 weeks $(\mathrm{p}=0.036)$ and 24 weeks $(\mathrm{p}<0.001)$. Among RF-negative patients, no significant difference was seen in ACR50 responses at 4, 12 or 24 weeks. Similar improvements in ACR20, 50, and 70 scores at Week 24 were observed following retreatment (Figure 3A) as well as an enhanced response in RF-positive patients compared to those who were RF-negative (Figure 3B). A smaller proportion of RF-positive patients (9\%) versus RF-negative patients (17\%) dropped out during the study. There was no appreciable difference in the percentage of patients with an ACR20, 50, or 70 response based on the reason for discontinuing their TNF inhibitor (lack of initial response, loss of response over time, tolerability).

When the results were evaluated by country, a greater percentage of Swedish patients achieved a 20\%, 50\%, and $70 \%$ improvement in the ACR score than patients from Canada (Table 3). This was mostly driven by the improvement in the acute-phase reactants (ESR and CRP; Table 4). Swedish patients started with higher inflammatory values and showed a greater improvement; while both cohorts showed similar decreases in the SJC and TJC. EULAR responses to RTX were good/moderate at 24 weeks in 87 patients $(72.5 \%)$ in the primary treatment period and in 57 patients $(74.0 \%)$ in the retreatment period. Clinical improvement was also seen in DAS28 scores. Mean reductions in DAS28 scores were seen as early as Week $4(-1.2 ; \mathrm{p}<0.001)$ and continued to improve at Weeks $24(-2.0)$ and $48(-2.1)$. A greater decrease in the DAS28 was observed in RF-positive patients versus RF-negative patients at Week 24 (Figure 4B). Consistent with the ACR response, a greater decrease in the DAS28 score was observed in RF-positive than in RF-negative patients ( -2.2 vs -1.4 at 24 weeks, respectively; Figure 4B). Reductions in DAS28 scores were observed during the retreatment period, at 12 weeks $(-2.3)$ and 24 weeks $(-2.2$; Figures $5 \mathrm{~A}$ and $5 \mathrm{~B})$. Swedish patients had a greater decrease in the DAS28 score compared to the Canadian population at Week 24 in the primary treatment period $(-2.4 \mathrm{vs}-1.9)$. As for the ACR responses, this factor was mostly driven by the greater improvement in the ESR. Outcomes at Week 24 following retreatment were similar, -2.2 versus -2.3 , respectively.

During the primary treatment period, clinically significant improvements in the HAQ-DI score were evident as early as Week $4(-0.2)$, with further improvements at Week 24 and at 48 weeks $(-0.4$ each). Slightly better improvement in the HAQ-DI score was observed in RF-positive compared to RF-negative patients $(-0.5$ vs -0.4 Week 24 primary treatment; -0.5 vs -0.3 Week 24 retreatment period). The proportion of patients with at least a 0.25 decrease in HAQ-DI score was $56.7 \%$ at 24 weeks after primary RTX treatment and $64.9 \%$ at 24 weeks after retreatment. The mean change from baseline in the FACIT-F score was statistically significant $(\mathrm{p}<0.001)$ at 12 weeks $(-7.3)$ and 24 weeks $(-9.1)$ after primary treatment. At 24 weeks after retreatment, the FACIT-F decreased -8.9 relative to the original baseline. A marked decrease in FACIT-F was observed in RF-positive patients compared with RF-negative patients following both the primary treatment and retreatment ( -10.1 vs -4.9 and -9.9 vs 4.9 , respectively).

The mean time to retreatment was 34 weeks or about 8.5 months. Based on information provided by the investigators, the decision to re-treat was most often based on clinical evidence of active disease, as indicated by DAS score, 26 patients (33.8\%); followed by SJC, 22 patients (28.6\%); and TJC plus SJC, 13 patients (16.9\%). The remaining 20.7\% of

Personal non-commercial use only. The Journal of Rheumatology Copyright @ 2011 . All rights reserved. 
A
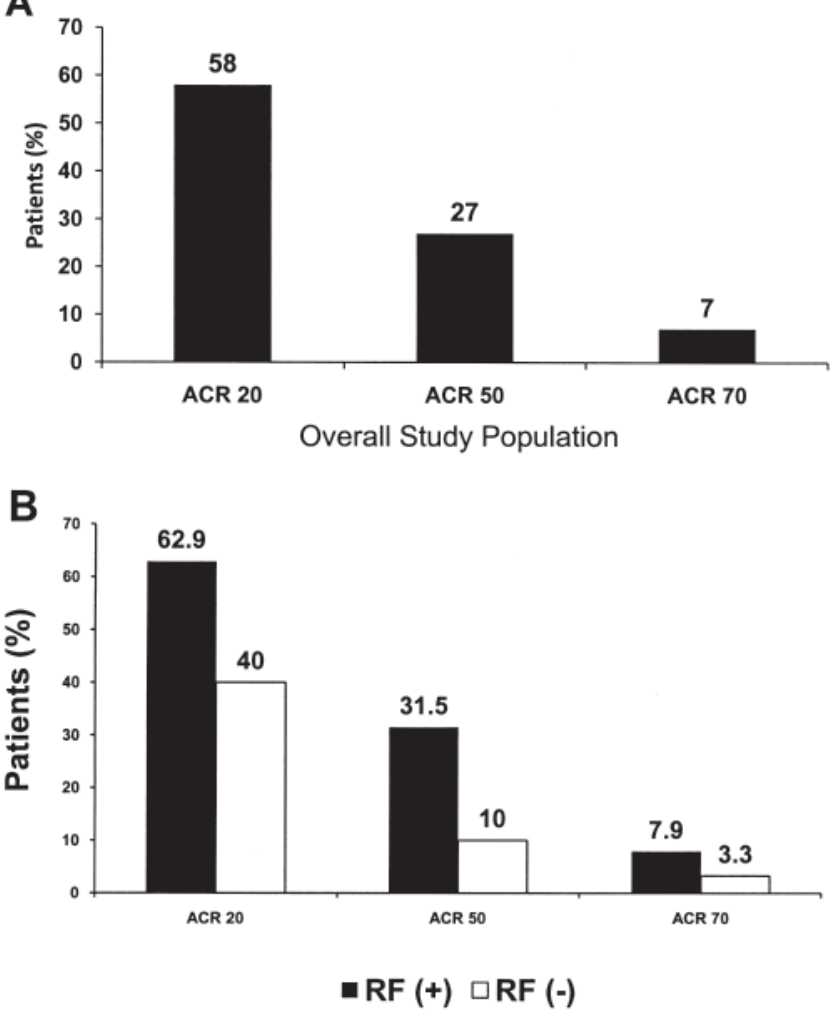

Figure 2. ACR response at Week 24 of primary treatment period for (A) overall study population, and (B) study population stratified by rheumatoid factor (RF) status.

patients re-treated based on the following: TJC alone, 6.5\%; fatigue, 2.6\%; MD global assessment of disease activity, 5.2\%; patient's global assessment of disease activity, $1.3 \%$; patient's global assessment of pain, $2.6 \%$; HAQ, $1.3 \%$; and other, $1.3 \%$.

\section{DISCUSSION}

To our knowledge, this is the first prospective interventional study that evaluated the safety and effectiveness of RTX in patients with RA who had discontinued their first TNF- $\alpha$ inhibitor and that was conducted in 2 countries with a comparable approach to the management of RA.

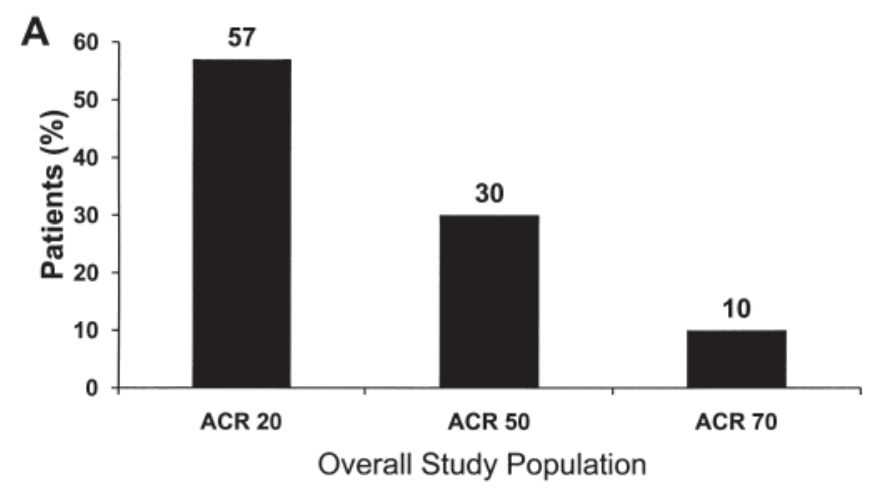

There is always a concern that the safety profile of a drug might be different from the one observed in randomized controlled trials (RCT) when tested in real-life clinical situations, where some patients might have not qualified for an RCT based on the inclusion/exclusion criteria. When compared to the REFLEX trial ${ }^{14}$, patients in our study had slightly less severe disease, measured by DAS28, CRP levels, and HAQ score, despite longer disease duration. Interestingly, the safety profiles were comparable during both the primary treatment period and the retreatment phase. No new signals were seen. RTX was well tolerated during the study, with no serious infusion reactions and a low incidence of serious infections. Fewer than $5 \%$ of study participants withdrew because of AE. About $10 \%$ of patients experienced SAE. No infections or infusion reactions were considered life-threatening. No clinically relevant laboratory abnormalities or vital sign changes were observed during the study period.

Regarding efficacy, comparable ACR responses and DAS28 improvement to the ones recorded in the REFLEX trial were also seen. A robust response was seen across all ACR core components, and improvements were seen in DAS28, ACR, and FACIT-F as early as 4 weeks after the first treatment course. The number of patients with an ACR50 response increased significantly after 12 and 24 weeks. Changes in the HAQ-DI and FACIT-F scores were consistent with the other RTX clinical outcome measurements by 24 weeks.

Our study was representative of a general RA population, with about $73 \%$ of the patients being RF-positive, and the response to RTX therapy was more pronounced in this subset compared to the RF-negative patients. However, a reasonable proportion $(40 \%)$ of the RF-negative patients achieved an ACR20 response, but only 10\% reached ACR50 at Week 24 . This enhanced response to RTX in seropositive patients is consistent with the current body of evidence from both clinical trials and registries ${ }^{14,19,20,21}$.

Important unanswered questions regarding repeat treatment with RTX are the issues of timing and the patient/physician decision process that leads to the initiation of subsequent courses. Interestingly, the average time to retreatment was

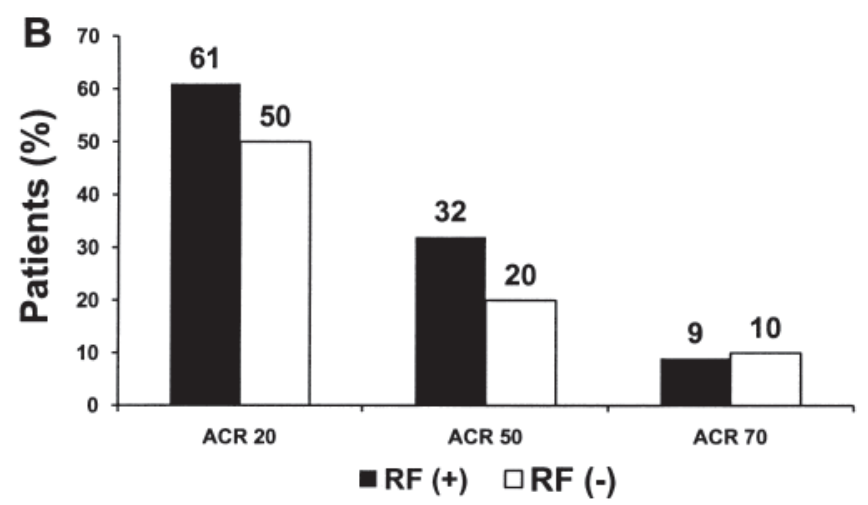

Figure 3. ACR response at 24 weeks of the retreatment period for (A) overall study population, and (B) study population stratified by rheumatoid factor (RF) status.

Personal non-commercial use only. The Journal of Rheumatology Copyright @ 2011 . All rights reserved. 
Table 3. American College of Rheumatology (ACR) response at Week 24 by country.

\begin{tabular}{lcc}
\hline ACR Response & Canada & Sweden \\
\hline 20 & $54 *$ & $69 *$ \\
50 & $55^{* *}$ & $77 * *$ \\
& $23 *$ & $38^{*}$ \\
70 & $30 * *$ & $31 * *$ \\
& $6 *$ & $10^{*}$ \\
& $9 * *$ & $13 * *$ \\
\hline
\end{tabular}

* Primary treatment period. ** Retreatment.

Table 4. Changes in Week 24 ACR core components.

\begin{tabular}{|c|c|c|c|}
\hline ACR Core Component & Canada & Sweden & $\begin{array}{c}\text { Overall Study } \\
\text { Population }\end{array}$ \\
\hline \multirow[t]{2}{*}{ SJC } & $-7.3 *$ & $-6.7 *$ & $-7.1 *$ \\
\hline & $-7.7 * *$ & $-7.5 * *$ & $-7.7 * *$ \\
\hline \multirow[t]{2}{*}{ TJC } & $-9.1 *$ & $-8.7 *$ & $-9.0^{*}$ \\
\hline & $-10.2 * *$ & $-7.5 * *$ & $-9.7 * *$ \\
\hline \multirow[t]{2}{*}{ MD global assessment of disease } & $-33.4 *$ & $-40.2 *$ & $-35.1 *$ \\
\hline & $-40.5 * *$ & $-45.6 * *$ & $-41.5 * *$ \\
\hline \multicolumn{4}{|l|}{ Patient global assessment } \\
\hline \multirow[t]{2}{*}{ of disease } & $-30.1 *$ & $-26.5^{*}$ & $-29.2 *$ \\
\hline & $-32.5 * *$ & $-22.4 * *$ & $-30.7 * *$ \\
\hline \multirow[t]{2}{*}{ Patient pain } & $-27.0 *$ & $-21.9 *$ & $-25.7 *$ \\
\hline & $-27.4 * *$ & $-23.0 * *$ & $-26.6^{* *}$ \\
\hline \multirow[t]{2}{*}{ HAQ-DI } & $-0.5 *$ & $-0.3 *$ & $-0.4^{*}$ \\
\hline & $-0.5 * *$ & $-0.3^{* *}$ & $-0.5 * *$ \\
\hline \multirow[t]{2}{*}{$\mathrm{ESR}, \mathrm{mm} / \mathrm{h}$} & $-14.3 *$ & $-20.6 *$ & $-16.0^{*}$ \\
\hline & $-16.4 * *$ & $-29.4 * *$ & $-18.7 * *$ \\
\hline \multirow[t]{2}{*}{$\mathrm{CRP}, \mathrm{mg} / \mathrm{dl}$} & $-14.7 *$ & $-20.1^{*}$ & $-16.1^{*}$ \\
\hline & $-12.2 * *$ & $-28.3 * *$ & $-15.3 * *$ \\
\hline
\end{tabular}

* Primary treatment period. ** Retreatment. SJC: swollen joint count; TJC: tender joint count; HAQ-DI: Health Assessment Questionnaire Disability Index; ESR: erythrocyte sedimentation rate; CRP: C-reactive protein.

about 8.5 months, slightly shorter than that reported in the longterm extension studies of 9 months ${ }^{16}$. The trigger to retreatment was a flare of disease based on the physician assessment of disease activity: one-third were based on the DAS28, but about $45 \%$ of physicians relied solely on the SJC or a combination of SJC and TJC, which reflects how physicians assess patients in usual practice rather than relying on validated composite assessment scores for this decision-making. This may be particularly relevant in established disease, where a composite measure may be influenced by underlying irreversible damage. Indeed, when the different components of the ACR response were analyzed separately (Figure 6), the least improvement was observed in the HAQ score, which can be easily affected by irreversible damage.

The better ACR responses and DAS28 improvement seen in the Swedish cohort compared to the Canadian patients was mostly driven by a more important reduction in the acute-phase reactants (ESR and CRP).

The response to a second course was also consistent with observed data from the extension of controlled trials and other open-label cohorts. Very few patients dropped out after receiving the second course $(6 / 77$, or $7.8 \%)$, strengthening prior observations that once a patient responds to a first course, clinical improvement is maintained with further retreatments. Of interest also is that 25 patients (20\%) maintained a good clinical response after the first course for at least 48 weeks and did not require retreatment within the pre-set observation period. The extended treatment-free interval (48 weeks) associated with a single course of RTX has been described by Edwards and colleagues ${ }^{9}$. The main difference between baseline characteristics in the single-course group versus the whole population was a higher proportion of RF-positive patients (88.0\% vs $74.2 \%)$. This is consistent with the finding of a more robust response in seropositive patients observed in our study as well as others. These observations, coupled with the fact that the average time between the first and second course is about 8.5 months, and the favorable efficacy and safety profile, make RTX a highly cost-effective and attractive therapy when a single TNF- $\alpha$ inhibitor has failed.

\section{APPENDIX}

List of principal investigators of the RESET Trial Group: Dr. C. Atkins, Dr. A. Beaulieu, Dr. W. Bensen, Dr. L. Bessette, Dr. G. Boire, Dr. M. Bokarewa, Dr. A. Bookman, Dr. T. Bracin, Dr. V. Bykerk, Dr. D. Choquette, Dr. A. Chow, Dr. H. El-Gabalawy, Dr. W. Fidler, Dr. M. Gagne, Dr. B. Haraoui, Dr. M. Hazeltine, Dr. H. Hellstrom, Dr. L. Jacobsson, Dr. N. Jones, Dr. A. Jovaisis,
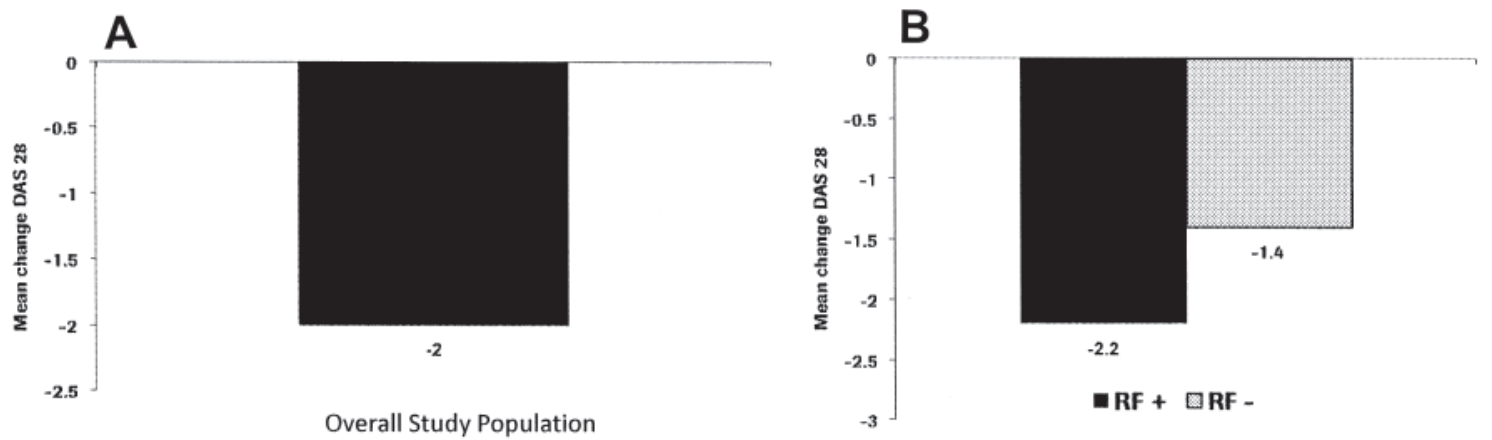

Figure 4. A. Mean changes in 28-joint Disease Activity Score (DAS28) at Week 24 (primary treatment) for the overall study population. B. Stratified by rheumatoid factor (RF) status. 

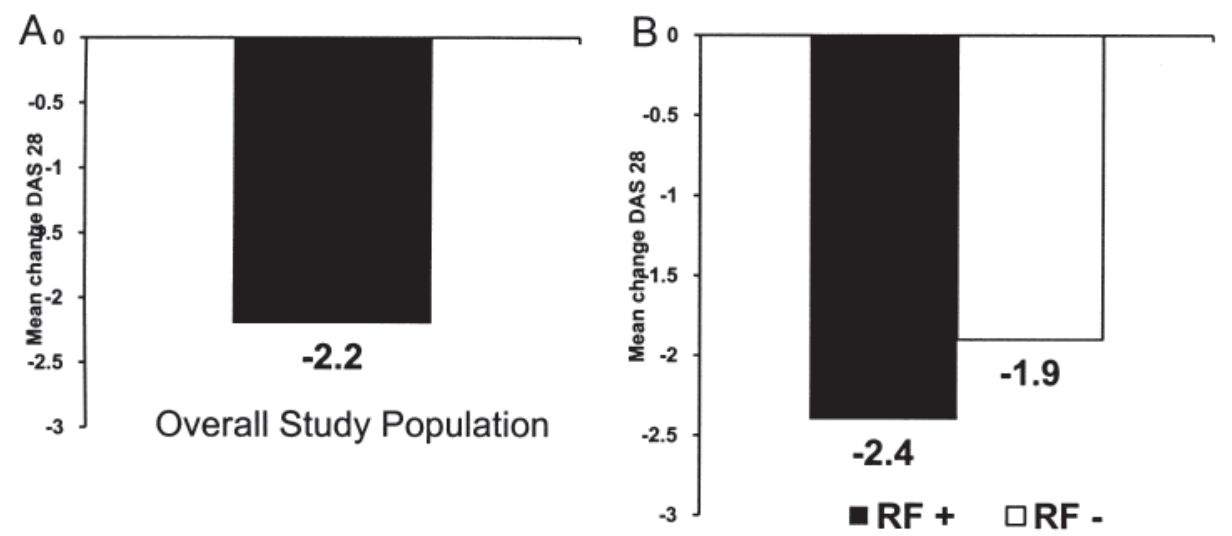

Figure 5. Mean changes in 28-joint Disease Activity Score (DAS28) at Week 24 following retreatment. A. Population as a whole. B. Stratified by rheumatoid factor (RF) status.

Dr. A. Karasik, Dr. J. Karsh, Dr. M. Khraishi, Dr. B. Moeller, Dr. F. Morin, Dr. S. Pillersdorf, Dr. J. Rodrigues, Dr. A. Russell, Dr. S. Seigel, Dr. K. Svensson, Dr. A. Teleman, Dr. E. Theander, Dr. S. Transoe, Dr. J. Waltin-James, Dr. A. Zickert, Dr. M. Zummer.

\section{REFERENCES}

1. Firestein GS. Etiology and pathogenesis of rheumatoid arthritis. In: Ruddy S, Harris ED, Sledge CB, editors. Kelley's textbook of rheumatology. Philadelphia: WB Saunders Company; 2001.

2. Pincus T, Callahan LF. Reassessment of twelve traditional paradigms concerning the diagnosis, prevalence, morbidity and mortality of rheumatoid arthritis. Scand J Rheumatol Suppl 1989;79:67-96.

3. Hochberg MC, Lebwohl MG, Plevy SE, Hobbs KF, Yocum DE. The benefit/risk profile of TNF blocking agents: Findings of a consensus panel. Semin Arthritis Rheum 2005;34:819-36.

4. Burmester GR, Monteagudo I, Saez I, Malaise MG, Canas da Silva J, Webber DG, et al. Adalimumab (Humira ${ }^{\circledR}$ ) is effective in patients who have previously been treated with TNF antagonists (etanercept and/or infliximab) in widespread clinical practice: 12 week outcomes in the REACT trial. Ann Rheum Dis 2005;64 Suppl 3:423.

5. Emery P, Keystone E, Tony H-P, Cantagrel A, van Vollenhoven R, Sanchez A, et al. IL-6 receptor inhibition with tocilizumab improves treatment outcomes in patients with rheumatoid arthritis refractory to
anti-TNF biologics: results from a 24-week multicenter randomised placebo controlled trial. Ann Rheum Dis 2008;67;1516-23.

6. Genovese MC, Becker J-C, Schiff M, Luggen M, Sherrer Y, Kremer $\mathrm{J}$, et al. Abatacept for rheumatoid arthritis refractory to tumor necrosis factor $\alpha$ inhibition. N Engl J Med 2005;353:1114-23.

7. Smolen J, Kay J, Doyle MK, Landewé R, Matteson EL, Wollenhaupt $\mathrm{J}$, et al. Golimumab in patients with active rheumatoid arthritis after treatment with tumour necrosis factor alpha inhibitors (GO-AFTER study): a multicenter, randomised, double-blind, placebo-controlled, phase III trial. Lancet 2009;374:210-21.

8. Rituxan (rituximab) product monograph. Hoffmann-La Roche Canada Ltd. June 2010.

9. Edwards JC, Szczepanski L, Szechinski J, Filipowicz-Sosnowska A, Emery P, Close DR, et al. Efficacy of B-cell-targeted therapy with rituximab in patients with rheumatoid arthritis. N Engl J Med 2004;350:2572-81.

10. Emery P, Fleischmann R, Filipowicz-Sosnowska A, Schechtman J, Szczepanski L, Kavanaugh A, et al. The efficacy and safety of rituximab in patients with active rheumatoid arthritis despite methotrexate treatment. Arthritis Rheum 2006;54:1390-400.

11. Rubbert-Roth A, Tak PP, Zerbini C, Tremblay J-L, Carreno L, Armstrong G, et al, on behalf of the MIRROR Trial Investigators. Efficacy and safety of various repeat treatment dosing regimens of

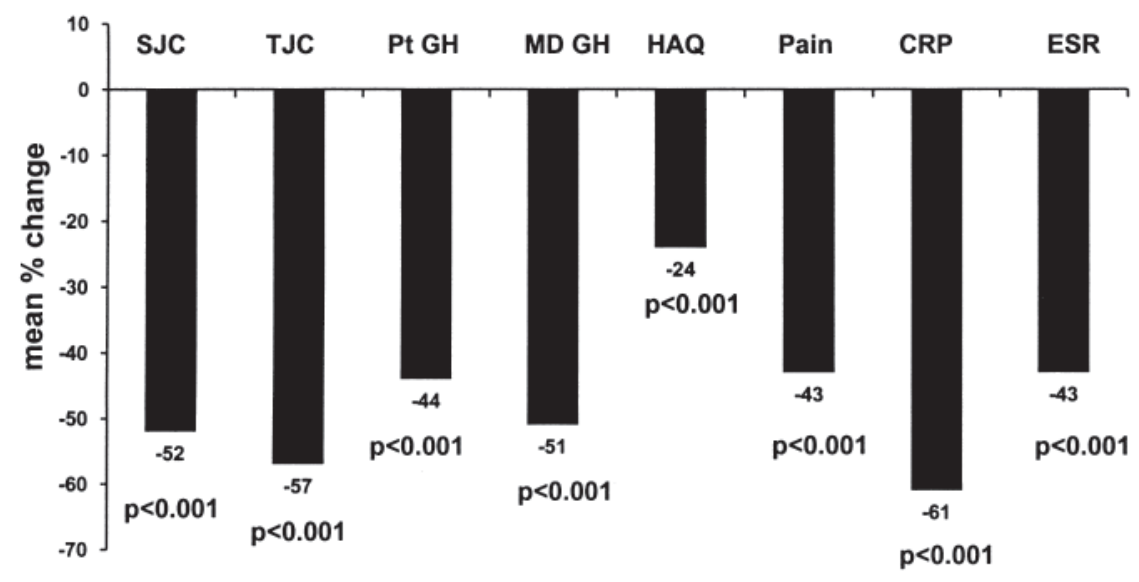

Figure 6. Changes in ACR core components at 24 weeks after primary rituximab treatment for the overall study population. SJC: swollen joint count; TJC: tender joint count; Pt GH: patient's global assessment of disease activity; MD GH: physician's global assessment of disease activity; HAQ: Health Assessment Questionnaire; CRP: C-reactive protein; ESR: erythrocyte sedimentation rate. 
rituximab in patients with active rheumatoid arthritis: results of a phase III randomized study (MIRROR). Rheumatology 2010;49:1683-93.

12. Emery P, Deodhar A, Rigby WF, Isaacs JD, Combe B, Racewicz AJ, et al. Efficacy and safety of different doses and retreatment of rituximab: a randomized, placebo-controlled trial in patients who are biological naïve with active rheumatoid arthritis and an inadequate response to methotrexate (Study Evaluating Rituximab's Efficacy in MTX iNadequate rEsponders; SERENE). Ann Rheum Dis 2010;69:1629-35.

13. Tak PP, Rigby WF, Rubbert-Roth A, Peterfy CG, van Vollenhoven RF, Stohl W, et al; for the IMAGE Investigators. Inhibition of joint damage and improved clinical outcomes with rituximab plus methotrexate in early active rheumatoid arthritis: the IMAGE trial. Ann Rheum Dis 2011;70:39-46.

14. Cohen SB, Emery P, Greenwald MW, Dougados MR, Furie RA, Genovese MC, et al. Rituximab for rheumatoid arthritis refractory to anti-tumour necrosis factor therapy. Arthritis Rheum 2006;54:2793-806

15. van Vollenhoven RF, Emery P, Bingham CO III, Keystone EC, Fleishmann RM, Furst DE, et al. Long-term safety of rituximab: follow-up of the RA clinical trials and retreatment population. Ann Rheum Dis 2010;69 Suppl 3:66.

16. Keystone E, Fleischmann R, Emery P, Furst DE, van Vollenhoven R, Bathon K, et al. Safety and efficacy of additional courses of rituximab in patients with active rheumatoid arthritis. An open-label extension analysis. Arthritis Rheum 2007;56:3896-908.
17. Keystone EC, Fleischmann RM, Emery P, Dougados M, Williams S, Linnik MD, et al. Multiple courses of rituximab produce sustained efficacy in patients with rheumatoid arthritis with an inadequate response to one or more TNF inhibitors [abstract]. Arthritis Rheum 2010;62 Suppl:S133.

18. Guidance for industry clinical safety data management. Definitions and standards for expedited reporting, ICH Topic E2A. Health Canada. [Internet. Accessed Aug 18, 2011.] Available from: http://www.hc-sc.gc.ca/dhp-mps/prodpharma/applic-demande/ guide-ld/ich/efficac/e2a-eng.php

19. Cohen S, Keystone E, Genovese MC, Emery P, Peterfy C, Tak PP, et al. Continued inhibition of structural damage over 2 years in patients with rheumatoid arthritis treated with rituximab in combination with methotrexate. Ann Rheum Dis 2010;69:1158-61.

20. Isaacs JD, Olech E, Tak PP, Deodhar A, Keystone E, Emery P, et al. Autoantibody-positive rheumatoid arthritis patients have enhanced clinical response to rituximab when compared with seronegative patients [abstract]. European League Against Rheumatism 2009 Annual European Congress of Rheumatology; Rome, Italy. Abstract 0256.

21. Mariette X, Kivitz A, Isaacs J, Stohl W, Tak PP, Jones R, et al. Effectiveness of rituximab (RTX) + methotrexate (MTX) in patients (pts) with early active rheumatoid arthritis (RA) and disease characteristics associated with poor outcomes [abstract]. Arthritis Rheum 2009;60 Suppl:1687. 